Résumé :

La récente publication de L'Histoire mondiale de la France de Patrick Boucheron a réactualisé un débat sur la mondialité de la culture et de l'histoire française. Ses pourfendeurs reprochent à cette vision élargie de l'Histoire française de ne pas tenir compte de certains socles de l'Histoire nationale. La question de fond qui est ici négociée en est une autre : Quel est le statut de la France dans la mondialisation ? Quel avenir pour l'exception française? Quelle place est accordée au roman national, forme de mise en narration pédagogique d'un pays héroïque ?

En effet, cet âpre débat est mené avec une ardeur nouvelle depuis la création d'un Ministère de l'Identité Nationale sous Nicolas Sarkozy en 2007 et le débat en ligne sur l'identité française qui s'ensuivait. Les écrivains réagissent depuis lors de multiples manières à ce débat, écrivant de la sorte un autre roman national. Alexis Jenni dans L'Art français de la guerre élucide les parts d'ombre des guerres coloniales, Jérôme Ferrari s'interroge dans Le Sermon sur la chute de Rome sur la pertinence même de la notion de déclin national, tandis que Magyd Cherfi dans Ma part de Gaulois relate sa vision du narratif national depuis sa perspective de fils d'immigrés.

\title{
Le roman national à l'épreuve du littéraire : Alexis Jenni, Jérôme Ferrari, Magyd Cherfi
}

Depuis plus de vingt ans, la notion de roman national est omniprésente dans les débats francofrançais autour de l'école et de l'enseignement de l'histoire. Si le terme roman national a émergé d'abord dans le débat historiographique, nous cherchons à donner à cette notion une tonalité différente dans la mesure où dans le roman national, il y a bien la notion de roman. Ainsi, il s'agit d'explorer trois romans esthétiquement très différents des années 2011-2016, lesquels prennent position de manière explicite par rapport à la question de l'identité française et de sa mise en narration. Ces romans sont L'Art français de la guerre d'Alexis Jenni, grande fresque romanesque présentant les parts d'ombre du colonialisme français, Le Sermon sur la chute de Rome de Jérôme Ferrari, roman questionnant la notion d'enracinement, ainsi que Ma part de Gaulois de Magyd Cherfi, dont le titre, au vu du nom peu gaulois de l'écrivain, est riche en potentialités sémantiques. Le but de cet article consiste à montrer comment la littérature cherche à proposer un autre récit collectif et à discuter les modalités par lesquelles les faits historiques qui dérangent sont rétablis dans le cadre d'une narration nationale alternative. Les trois auteurs, en dépit de leur différences esthétiques ont été retenues car outre leur travail littéraire, tous les trois interviennent régulièrement dans le débat public sur des thèmes liés au débat sur l'identité nationale. Ainsi, Jenni publia en 2016, un long dialogue avec Benjamin Stora, Les mémoires dangereuses, dans lequel ils s'interrogent sur les répercussions de la guerre d'Algérie dans la France contemporaine. Jérôme Ferrari a tenu pendant quelques semaines une chronique dans La Croix dans laquelle il explicite ses positions politiques exprimées, de façon plus discrète, 
dans ses romans. Cherfi, quant à lui, intervient régulièrement dans Libération et ses textes s'interrogent avec acuité sur ce qu'être Français issu de l'immigration veut dire.

Dans un premier temps, il convient de s'attarder quelque peu sur la notion de roman national, débattue de manière féroce depuis des années. La publication de l'Histoire mondiale de la France de Patrick Boucheron en janvier 2017 déclencha une discussion de grande envergure autour de cette œuvre. Le titre en indique la conception, explicitée par l'équipe éditoriale dès la préface :

Expliquer la France par le monde, écrire 1'histoire d'une France qui s'explique avec le monde: tout 1 'effort vise en somme à défaire la fausse symétrie de la France et du monde.

La France n'existe pas séparément du monde, le monde n'a jamais la même consistance pour la France. [...] Mais, à différents moments de l'histoire longue des mondialisations, dans les rapports changeants entre ce qui se donne comme „France“ et ce qui s'appréhende systématiquement comme „monde“ surgissent d'autres configurations sociales, des filiations multiples, des bifurcations inattendues, une histoire décalée, bref, une histoire en mouvement. (Boucheron 12)

Il s'agit donc de montrer l'enchevêtrement de l'histoire nationale avec celle des autres pays, de mettre en exergue comment la France dans son évolution historique n'existe pas en autarcie mais bien comment elle instaure, à travers les siècles, un dialogue avec le reste du monde. En effet, le projet est ambitieux à plus d'un titre. Il réunit une équipe de 147 historiens ayant rédigé des chapitres consacrés à une grande variété d'événements-clé de l'histoire française allant de l'homme de Cro-Magnon jusqu'aux événements du Bataclan en novembre 2015. À chaque événement présenté est consacré un chapitre de trois ou quatre pages enrichies de quelques références bibliographiques permettant de pousser plus loin l'analyse. Les chapitres regroupent ce que l'équipe de chercheurs autour de Véronique Porra (Porra et alii) a nommé "lieux d'oubli" de la Francophonie en ce qu'ils mettent en lumière autant de chapitres de l'histoire française oubliés ou, le cas échéant, immédiatement incorporés dans le grand récit national sans questionner leur potentiel subversif. Ces chapitres sont la fondation du Musée d'histoire naturelle dans le cadre du projet jacobin en 1793, la fondation du Comité philhellène en 1825, l'arrivée des parents de Charles Aznavour à Marseille juste un an avant la naissance de celui qui deviendra un des plus grands chanteurs de la chanson française. La Débâcle de 1940 est présentée à travers la découverte fortuite de la grotte de Lascaux, la décolonisation est représentée par la publication du texte Les damnés de la terre de Frantz Fanon,.

La tare principale de cette nouvelle Histoire résiderait, selon Alain Finkelkraut, dans le fait qu'elle manque de mentionner les grands auteurs de l'Histoire littéraire française. Ce reproche, 
étonnant à plus d'un titre, évoque en effet la séparation de l'Histoire et de l'Histoire littéraire au $\mathrm{XIX}^{\mathrm{e}}$ siècle sur laquelle il sera bon de revenir.

Les réactions à 1'Histoire mondiale de la France font penser immédiatement à celles qu'a suscitées le manifeste Pour une littérature monde en français de Jean Rouaud et de Michel Le Bris. En effet, les deux textes s'inscrivent dans le contexte d'une campagne électorale caractérisée en 2007 comme en 2017 par l'émergence d'un candidat d'un nouveau type (Nicolas Sarkozy et Emmanuel Macron) et les deux cherchent à renégocier, respectivement par le biais de la littérature et de l'Histoire la "mondialité" de la France et le statut de la Francophonie. Désormais dans l'univers de la culture, à l'instar de la scène politique, deux camps semblent s'opposer, transcendant d'ailleurs les clivages politiques classiques: les mondialistes (qui reconnaissent, grosso modo, que la mondialisation est un phénomène inévitable auquel il faut répondre par des moyens littéraires et culturels t soulignant l'importance de la Francophonie et de la migration) versus les enracinés (plus parisiano-centristes, axés sur la littérature pure et la prédominance du style). Cette toile de fond permet de mieux saisir les subtilités idéologiques du billet suivant, de Jean Pierre Rioux s'inscrivant également en faux contre l'Histoire mondiale.

Autrement dit, cette «Histoire mondiale de la France » est bien de son temps. Elle flotte même fort bien dans l'air du temps, celui de l'individualisation de la consommation d'histoire; du joyeux ou désespéré jeu de quille face à tout ce qui est institué, dogmatique, épousant une ambition «politique » et civique, qu'elle soit progressiste ou conservatrice. Mais aussi d'une certaine myopie des élites du savoir à l'heure (voir le sondage Ifop-Synopia publié par «La Croix » le 14 mars) où, dramatiquement mais clairement, les Français n'arrivent plus à dire «nous » tout en conservant à $86 \%$ un fort sentiment d'appartenance à la nation française et prennent fort peu en considération, semble t-il, ce «global» et ce «multi » dont seule une frange élitaire est pénétrée. (Jean-Pierre Rioux 2017)

On l'a compris, la cible de sa critique est moins l'Histoire mondiale de la France que le «mondialisme» des élites, et dans un contexte fortement électoral, l'orientation internationale du candidat Emmanuel Macron avec son discours (souvent prononcé en anglais ou en franglais), construit autour de la start-up nation, de l'ouverture, de la mondialisation comme une chance pour la France. Ce faisant, il a, tel Nicolas Sarkozy en 2007, déstabilisé le consensus social-colbertiste et anti-américain qui régnait en France depuis la Deuxième Guerre Mondiale. Ainsi, l'Histoire mondiale de la France serait l'emblème d'un nouveau monde, promis par le candidat trentenaire.

\section{Le roman national}


$\mathrm{Au}$ fond, la question que fait surgir l'Histoire mondiale de la France revient à savoir comment relater l'histoire de la nation. Il existe en effet une tradition d' historiographie nationale, populaire et pédagogique moins axée sur la vérité historique au sens strict du terme que sur l'aspect pédagogique, sur le fait d'inculquer une certaine fierté nationale aux jeunes générations. Ceci trouve son incarnation contemporaine dans des émissions de télévision comme Secrets d'histoire animée par Stéphane Bern ou des succès en librairie comme Métronome par Lorànt Deutsch, épinglés tous les deux par des historiens comme Suzanne Citron ou William Blanc. Il existe une véritable mythologie nationale de la nation unie, dotée d'un génie proprement français que l'on peut retracer jusqu'au début de l'historiographie française au XIX ${ }^{\mathrm{e}}$ siècle, véhiculée par des manuels comme le Lavisse. Nicolas Offenstaedt, historien spécialiste du « roman national » le définit comme suit :

L'expression " roman national », popularisée par Pierre Nora, est passée dans le langage courant : elle désigne le récit patriotique, centralisateur, édifié par les historiens du $\mathrm{XIX}^{\mathrm{e}}$ siècle tout à la louange de la construction de la nation. Le récit national met en avant la grandeur du pays, ses hauts faits et édulcore souvent les pages plus délicates. Il naturalise le « patriotisme », depuis les temps anciens. Dans sa version mystique, la France existerait de toute éternité et les souverains qui se sont succédé n'auraient fait qu'accomplir une destinée quasi naturelle, transcendante. (Nicolas Offenstaedt, « À bas le roman national », L'Histoire, juillet-septembre 2009, http://www.lhistoire.fr/bas-le-roman-national)

La notion de roman national fut d'ailleurs popularisée par Pierre Nora dans sa postface aux Lieux de mémoire en 1995 ; en effet il y cite un ouvrage publié peu avant qui s'intitule Voyage au cour du malaise français. Anti-racisme et roman national par Marcel Yonnet, publié en 1993. Dans le cadre de ce que certains considèrent comme une droitisation du discours public en France, palpable depuis les années $1990^{1}$ mais ayant atteint son paroxysme provisoire avec l'instauration d'un ministère de l'identité nationale en 2007, cette notion, qui caractérise pourtant un récit hérité du XIX ${ }^{\mathrm{e}}$ siècle, fut utilisée par des politiciens et des historiens de droite afin de chercher à réinstaurer une forme de storytelling national dont émanerait une essence nationale. Elle était également présente dans la campagne électorale en 2017, durant laquelle les deux candidats de la droite se prononcèrent pour la réintroduction du roman national dans les programmes scolaires. Il faut savoir que la notion n'y a jamais officiellement figuré ; et, comme nous le rappelle Vincent Chambarlhac, la discussion autour du rôle que joue

\footnotetext{
${ }^{1}$ On peut mentionner deux publications se sont penchées sur cette question: Le numéro spécial des Yale French Studies, 116/117 (2009), « Turns to the Right ». Et : Noiriel, Gérard. À quoi sert l'identité nationale?. Paris: Agone, 2007.
} 
l'enseignement de l'historien dans la construction de la nation avec, omniprésente, l'ombre du déclin national qui hante la conscience nationale, est vieille comme la $\mathrm{V}^{\mathrm{e}}$ République.

Le déclin fait partie intégrante du récit collectif français, tellement la pensée française en est empreinte. La raison semble notamment résider dans une glorification de l'époque classique, pendant laquelle l'espace du politique et l'espace de la production culturelle coïncidaient à Versailles, créant de la sorte une dynamique culturelle particulière. Si le discours sur la déchéance française est une constante depuis le $\mathrm{XIX}^{\mathrm{e}}$ siècle, il n'en a pas moins pris une nouvelle envergure depuis quelques années avec l'émergence de certains penseurs dits néoréactionnaires fustigés par Daniel Lindenberg dès 2002 dans son ouvrage Le Rappel à l'ordre. Bien qu'il s'agisse d'un groupe hétéroclite à l'idéologie protéiforme, un examen rapide de leurs idées permet d'identifier un socle idéologique basé sur le complexe du village d'Astérix, constamment menacé par des forces extérieures obscures Le roman national serait donc la tentative d'endiguer tous ces maux et de pérenniser une histoire didactisée dont émanerait une essence, un génie national.

Pris au sens strict du terme, le terme roman national est un contresens et toutes les définitions, aussi divergentes soient-elles, pour définir le terme roman convergent sur un point : c'est d'une œuvre fictionnelle qu'il s'agit, c'est un texte qui simule la réalité, qui fait comme si (lat. fingere, faire comme si). L'ambition du roman national, serait de s'en tenir aux faits historiques s'étant réellement produits (même si ceux-ci sont parfois un peu édulcorés), sans « faire comme si ». En même temps, le roman historique (dont le grand paradigme est Walter Scott) permet une meilleure compréhension des raisons et des motivations des personnages qui portent les grands événements historiques :

Es kommt im historischen Roman nicht auf das Nacherzählen der goßen historischen Ereignisse an, sondern auf das dichterische Erwecken jener Menschen, die in diesen Ereignissen fungiert haben. Es kommt darauf an, nacherlebbar zu machen, aus welchen gesellschaftlichen und menschlichen Beweggründen die Menschen gerade so gedacht, gefühlt und gehandelt haben. ${ }^{2}$ (Lukács 37-38)

\footnotetext{
${ }^{2}$ Ce qui importe dans le roman historique, n'est pas un résumé des grands évènements, mais il s'agit de réveiller de manière poétique ces êtres humaines qui ont été impliqués dans ces événements. Il importe de faire comprendre, pour quelles raisons sociétales et humaines, les gens ont pensé, senti et agi comme ils l'ont fait. (Traduction de TO)
} 
En même temps, de nombreux historiens, tel que Haydn White dans sa magistrale étude Metahistory, ont souligné que l'Histoire opère avec les mêmes procédés narratifs que la littérature, et s'interrogent sur les modalités narratives avec lesquelles travaille l'historiographie tout en soulignant l'importance de la tradition inaugurée par Michelet. Avant que ne s'effectue la grande séparation entre les deux écritures, historique et littéraire, entérinée au XIX ${ }^{\mathrm{e}}$ siècle, leur modi operandi étaient relativement proches, comme nous le rappelle Ivan Jablonka dans son plaidoyer pour un renouvellement littéraire des sciences sociales par l'imagination littéraire :

Contre cette profession de foi néo-aristotélicienne, qui exclut l'histoire de la « littérature » au sens romantique, les historiens affirmeront qu'ils peuvent eux aussi être des créateurs. Non qu'ils inventent des êtres de fiction, mais en personnifiant des collectifs, en nommant des forces, ils font naître de nouveaux personnages. Chez Michelet, ce sera la France, le Peuple, la Sorcière, la Femme ; chez Ranke, l'Esprit du Temps, les Idées, les Puissances invisibles comme l'État, l’Église [....]. (Jablonka 60)

Parallèlement, Ivan Jablonka nous rappelle à quel point Histoire, Littérature et même Sciences étaient imbriquées encore à la fin du $\mathrm{XIX}^{\mathrm{e}}$ siècle, dans la mesure où Balzac se considérait plus comme un historien que comme un écrivain et Zola envisageait ses Rougon-Macquart comme un laboratoire confirmant ou infirmant les théories de Darwin sur l'hérédité, tandis que Michelet compte parmi les grands prosateurs de son temps. Il existe, entre l'Histoire et la Littérature, un énorme champ en jachère dont l'exploration sérieuse ne fait que commencer. C'est précisément dans cette zone indécise entre Histoire et Littérature que s'opère, selon nous, la renégociation du roman national. En effet, les écrivains réagissent de moult manières à ce nouveau discours dominant et s'attachent à créer autant de contre-discours qui déstabilisent le discours hégémonique des enracinés. Notre argumentation est basée sur la prémisse que les écrivains, à l'aune de ce débat qui allait rapidement s'envenimer, créent ainsi des espaces intermédiaires entre Histoire et Littérature permettant de la sorte de renouveler la définition du roman national.

\section{Alexis Jenni et les oublis de l'histoire}

Ainsi, L'Art français de la Guerre d'Alexis Jenni, considéré par la critique comme le Goncourt anti-identité nationale (Perraud 2012), élucide les oublis de l'histoire française : cruautés commises pendant les guerre d'Indochine et d'Algérie, liens inquiétants qui se tissent entre les acteurs des guerres coloniales comme la guerre d'Algérie et l'extrême-droite, rendez-vous manqués de la France avec les banlieues, autant de chapitres que le roman cherche à démêler. La structure de ce roman dédoublé relate l'histoire d'un jeune homme désireux de refaire sa vie après avoir divorcé de sa femme et avoir rompu avec son existence bourgeoise. Lors de ses 
déambulations, il fait la connaissance d'un peintre qui va lui raconter ses aventures pendant les guerres coloniales :

Il m'éclaira, Victorien Salagnon, le rencontrer aux creux de ma vie m'éclaira. Il me fit reconnaître ce signe qui parcourt l'Histoire, ce signe peu connu et pourtant visible, qui est toujours, qui est un rapport, qui est une fraction, qui s'exprime comme suit : dix pour un. Cette proportion est le signe souterrain du massacre colonial. (Jenni 29)

A l'instar de Max Aue, héros des Bienveillantes de Jonathan Littell, Salagnon est un héros « systématique », dans la mesure où il est présent dans tous les combats armés menés par la France après la Libération. Désireux de s'installer en Algérie au printemps 1945, il relatera le massacre commis par l'armée française contre les insurgés de Sétif, Khelma et Guerrata. Ensuite, il se bat en Indochine contre le Viet-Cong et ne cesse de dévoiler la violence et la brutalité (escamotées et absentes du débat public) de ces combats. En effet, le roman s'attache avant tout à en dépeindre la brutalité sans nom :

Des dizaines de corps descendaient la rivière, une réserve inépuisable de corps s'écoulait par la rivière, les femmes flottaient entourées de leurs cheveux étendus autour d'elle, les enfants pour une fois allaient sans brusqueries, les hommes se ressemblaient tous le pyjama noir qui sert d'uniforme à tout le pays.

[...] Plus haut, ils doublèrent un village incendié. Il fumait encore mais tout avait brûlé, le chaume des toits, les palissades de bambou, les cloisons de bois tressé. Il ne restait que des poutres verticales noircies et des tas fumants, entourés de palmiers étêtés et de cadavres de cochons. (Jenni 378-379)

À l'aune du contexte de publication, le débat autour de l'identité nationale qu'avait initié Nicolas Sarkozy en 2008-9, l'on n'est guère étonné de constater que cette question traverse le texte tel un fil rouge. Le narrateur, lors de ses balades nocturnes, s'interroge avec acuité sur la question de savoir ce qu'être Français veut dire face au débat surchauffé de la deuxième moitié des années 2010. En s’interrogeant sur les raisons de la crise identitaire traversée par le pays, il envisage celle-ci tout d'abord comme une crise de la narration collective, laquelle fait défaut depuis la mort du général de Gaulle :

Je pense à la France ; mais qui peut dire sans rire qu'il pense à la France ? Sinon les grands hommes et seulement dans leurs mémoires. Qui, sinon le général de Gaulle peut dire sans rire qu'il pense à la France. [...] Alors je parle de la France comme de Gaulle en parlait, en mélangeant les personnes, en mélangeant les temps, confusant la grammaire pour brouiller les pistes.

De Gaulle est le plus grand menteur de tous les temps, mais menteur il l'était comme mentent les romanciers. Il construisit par la force de son verbe, pièce par pièce, tout ce dont nous avions besoin pour habiter le XXe siècle. Il nous donna, parce qu'il les inventa, les raisons de vivre ensemble et d'être fiers de nous. Et nous vivons dans les ruines de ce qu'il construisit, dans les pages déchirées d'un roman qu'il écrivit, que nous prîmes pour une encyclopédie, que 
nous prîmes pour l'image claire de la réalité alors qu'il ne s'agissait que d'une fiction, une fiction en laquelle il était doux de croire. (Jenni 160=161)

Ce passage prend la mesure de l'ampleur de l'entreprise romanesque d'Alexis Jenni et ses contradictions. S'il reconnaît l'importance de la grande geste gaullienne, de son roman national, pour la cohésion du pays, force lui est admettre également que ce grand récit collectif est basé sur un mensonge. C'est ainsi qu'apparaît toute l'ambigüité du récit collectif qui n' est pas indispensable pour pouvoir vivre au sein de ce que Anderson nomme une «communauté imaginaire ». Or la vérité ne tarde pas à éclater au grand jour, la fiction du général escamotant autant de souffrances, d'histoires, d'injustices qui allaient se faire entendre. La définition de la nation qui surgit, en filigrane, dans ce passage, est celle d'une communauté partageant non pas le même sang, ni même un territoire, mais comme une communauté narrative, partageant une même histoire, une même fiction. Qu'advient-il à la nation dès lors que ces grands récits s'avèrent des leurres? Davantage que la narration, c'est la langue qui véhicule cette narrationporteuse d'identification également problématique dans le contexte de la mondialisation:

La France est l'usage du français. La langue est la nature où nous grandissons; elle est le sang que l'on transmet et qui nous nourrit, Nous baignons dans la langue et quelqu'un a chié dedans. Nous n'osons plus ouvrir la bouche de peur d'avaler de ces étrons de verbe. Nous nous taisons. Nous ne vivons plus. [...] On rêve d'utiliser l'anglais qui ne nous concerne pas. (Jenni 197-198)

Voici une interprétation intéressante de l'anglomanie française : traditionnellement ancrés dans la langue nationale, les débats vifs et riches qui la caractérisaient se sont appauvris, standardisés, jusqu'à en devenir monotones, insignifiants. Le rouleau-compresseur de la mondialisation avec son «il n'y a pas d'alternative » a eu raison de la culture des débats, jadis si vive. Et si on n'a plus rien à se dire, on peut facilement passer à la langue de l'Empire.

Jérôme Ferrari - Pour une mémoire palimpseste

De façon moins didactique, deux romans de Jérôme Ferrari dépeignent les contradictions et les blancs de l'histoire française. Dans Où j'ai laissé mon âme l'auteur explore la thématique épineuse de la torture pendant la guerre d'Algérie en l'inscrivant dans l'Histoire européenne avec sa grande hache, comme l'a formulé Perec. Car le tortionnaire est lui-même victime de la torture subie à Buchenwald. 
En ce qui concerne Le Sermon sur la Chute de Rome, le parallèle entre la France et l'empire romain a été tiré à bien des reprises : la France, c'est la nouvelle Rome, puisque, moyennant un processus de translatio imperii, elle a su mener à bien la mission civilisatrice de Rome. ${ }^{3}$

Organisé autour d'une triple temporalité, le roman contient d'abord des éléments du discours de Saint-Augustin lorsqu'il apprend que les Barbares ont bien pris Rome. L'analogie avec la France d'aujourd'hui est vite établie. Il convient de noter cependant que ce roman remet en cause cette idéologie basée sur l'analogie dans la mesure où le discours d'Augustin met en exergue justement le fait qu'une culture ne meurt pas ainsi et qu'il en reste inévitablement des traces, matérielles, linguistiques, culturelles.

La deuxième temporalité est fournie par l'histoire de Marcel Antonetti, grand-père de Matthieu, l'un des protagonistes que l'on voit représenté sur une photo dans une colonie française :

Il était entouré de sa femme et de ses enfants, l'ancre dorée des troupes coloniales ornait ses manches et son képi, mais l'influence toxique de sa terre natale le renvoyait de nouveau à ce qu'il n'avait jamais cessé d'être, un paysan inculte et gauche que le destin avait envoyé dans un monde qu'il ne méritait pas (Ferrari 70)

Et quand Marcel commence ses fonctions au cadastre colonial, il est vite confronté à un univers du monde colonial en pleine déchéance. La descente aux enfers est mise en valeur par le lexique de la pourriture et de la déchéance:

Il prit ces fonctions de rédacteur dans les bureaux de l'administration centrale d'une grande ville en Afrique qui ressemblait davantage à un invraisemblable amas de taudis et de boue qu'à une ville qu'il aurait pu rêver, car le monde persistait à contrarier ses rêves au moment même où ils devenaient réels. Dans les rues, les parfums étaient tellement violents que même les fruits mûrs et les parfums semblaient exhaler des suavités délétères de putréfaction, il réprimait constamment des nausées en déambulant dans la dignité de son costume de lin au milieu des hommes et des bêtes parmi lesquels flottaient des effluves de chairs exotiques et sauvages portés par le froissement des tissus de couleur et la proximité des indigènes lui répugnait chaque jour davantage, il n'était pas venu leur apporter une civilisation qu'il n'avait lui-même que connue de loin et par ouï-dire dans la voix de ses maîtres mais encaisser une dette ancienne dont le paiement avait été si longtemps différé, il était venu pour vivre la vie qu'il méritait et qui n'avait cessé de se dérober à son étreinte1. (Ferrari 132-133)

Nous voyons à quel point le projet colonial, avec son mot d'ordre la mission civilisatrice, était d'emblée voué à l'échec car ses dépositaires étaient à peine plus éduqués que les «indigènes » Le vocabulaire de la déréliction qui traverse le texte révèle à quel point l'échec du projet

\footnotetext{
${ }^{3}$ La notion de translatio imperii a été théorisée par Jacques Le Goff, désignant la migration de l'Est vers l'Ouest des centres du pouvoir. Concernant la France en tant que nouvelle Rome, cette pensée semble être très présente auprès d'une certaine droite française: Éric Zemmour. Mélancolie française. Paris, Albin Michel. 2010.
} 
colonial lui était inhérent. En revanche, le petit fils Matthieu vivra pleinement le rêve français : après des études scolaires passablement réussies, il s'inscrira en Sorbonne pour y suivre des études de philosophie, discipline la plus française de toutes. Son désenchantement est radical lorsqu'il doit se rendre à l'évidence que le rêve français est en train de s'essouffler :

Mais l'ambiance de dévotion qui régnait dans la salle poussiéreuse de l'escalier $\mathrm{C}$ où on les avait relégués ne pouvait dissimuler l'ampleur de leur déroute, ils étaient tous des vaincus, des êtres inadaptés et bientôt incompréhensibles, les survivants d'une apocalypse sournoise qui avait décimé leurs semblables et mis à bas les temples qu'ils adoraient [...] (Ferrari 59)

Troisième temporalité du roman: Matthieu, désenchanté par l'univers de l'Université, amoureux de la Corse et très proche de son ami Libero, décide de prendre en charge un bar en Corse lequel avait connu plusieurs échecs auparavant. Dans un premier temps, ils semblent merveilleusement bien réussir leur pari car ils réussissent à transformer un bar quelque peu miteux en un véritable lieu de rencontre huppé et mondialisé, symbole de ce nouveau monde cher à certains de nos dirigeants. Or même le bar en Corse, meilleur des mondes possibles, échoue à la fin de la narration. Reste les vieilles pierres de Hippone et la sagesse de SaintAugustin : les mondes périssent et renaissent. Comme nous le rappellent Claudia Jünke (2016 et 2018) et Timo Obergöker (2018) ce roman constitue avec son prédécesseur Où j'ai laissé mon âme, un exemple éloquent pour ce que le chercheur britannique Max Silverman a appelé dans son ouvrage éponyme la mémoire-palimpseste (palimpsestic memory). Il constate que les grands traumatismes du $\mathrm{XX}^{\mathrm{e}}$ siècle ne constituent nullement des évènements isolés mais que ceux-ci sont interconnectés par des fils souvent ténus.

I have chosen the term palimpsestic memory to discuss this hybrid form because, of all the figures which connect disparate elements through a play of similarity and difference (analogy, metaphor, montage and so on), the palimpsest captures most completely the superimposition and productive interaction of different inscriptions and the spatialisation of the time central to the work which I would like to highlight. (Silverman 5)

Des liens ténus s'établissent ainsi entre les différents moments de l'Histoire nationale, des liens souvent inattendus qui montrent à quel point l'histoire coloniale et celle de la Seconde Guerre Mondiale et de la Shoah sont enchevêtrées, que la folie de Stalingrad fut préparée dans le gaz d'Ypres. La perspective historique varie, elle aussi, en fonction de l'endroit où l'on réside. Si le 8 mai en Europe fut célébré comme la journée de la libération du joug nazi, il est commémoré en Algérie comme la journée du massacre de Sétif, inaugurant d'une certaine façon la guerre d'Algérie. Eu égard aux connexions qui s'établissent entre les différents événements, tout roman basé sur la chronologie paraît anodin. 


\section{Magyd Cherfi - Comment peut-on être Gaulois ?}

Ainsi n'est-il pas étonnant si les écrivains que l'on subsume dans la catégorie fourretout « issus de l'immigration »s'interrogent dans leur travail avec une acuité particulière sur l'importance de créer un récit national susceptible d'inclure les immigrés récents, ceux qu'un récit exclusivement européen risque d'ostraciser. Un exemple de texte autofictionnel qui cherche à inclure les voix immigrées dans un plus ample panorama national est Ma part de Gaulois de Magyd Cherfi, le chanteur du groupe Zebda.. Un rapide examen du paratexte, en l'occurrence de la couverture, nous permet de prendre la mesure de l'ironie dont use l'auteur afin de lancer un défi au lecteur potentiel. Le mot Gaulois est riche en connotations ; en effet dans l'historiographie populaire et légendaire comme la pratiquait le Lavisse, les Gaulois sont les ancêtres directs des Français, habitant la Gaule qui changea ensuite de nom pour devenir, au Moyen-Âge, la France. Comme nous le rappelle Suzanne Citron, les Gaulois représentent une nation hétérogène aux contours flous et ils ne peuvent en aucun cas être envisagés comme des proto-Français.

Par le mot «pays », par celui de « France », hier et aujourd'hui sont confondus. Recouvrant un présent réel, ces mots projetés dans un autrefois de légende, suscitent l'imaginaire d'un pays déjà là. L'histoire de France commence par le mythe de France. (Citron 36)

La photo qui orne la couverture du livre montre l'idylle familiale d'une famille immigrée, vraisemblablement nord-africaine, dans les années 1970/1980. Le nom peu gaulois de l'écrivain renforce cette impression. La Gaule, aussi fantasmagorique fût-elle, est bien loin dans cet ensemble. Dès la couverture, la question centrale du texte est abordée : quelle est la place d'un Autre dans une communauté imaginaire ? Car, si on s'en tenait à une définition purement ethnique de la francité, l'auteur-narrateur ne trouverait pas sa part dans le projet national. Nous verrons comment il parvient à définir «sa part de gaulois». Le récit se déroule dans les banlieues toulousaines au début des années 1980. Magyd est un jeune collégien amoureux des livres et de littérature, fait qui le rend suspect aux yeux de ses amis qui le taxent de «pédé ». Cette situation d'outsider, douloureuse et compliquée pour Magyd, lui confère néanmoins une position depuis laquelle il est en mesure de porter un regard critique sur sa communauté nordafricaine et sur les Français non-immigrés. Car incontestablement, par le biais de ses lectures, sa réussite scolaire et son amour des mots, le narrateur réalise le «rêve français », défini par Peter Gumbel dans les termes suivants : 
Les Français se moquent souvent des Américains et de leur «American dream », cette grande vision d'un pays dans lequel tout est possible et où les possibilités se rencontrent à chaque coin de rue. Mais la France a aussi son propre «French dream ». Il s'appelle l'école.

L'école est bien plus qu'un lieu où les enfants viennent apprendre: dans l'Hexagone, elle incarne les valeurs les plus chères à la société. Il est impossible d'ignorer le sentiment de fierté nationale qui repose sur le fait que l'école est gratuite et laïque. Impossible aussi de ne pas être impressionné par l'idéal méritocratique selon lequel n'importe quel enfant, issu de n'importe quel milieu, peut, en théorie, intégrer les plus hautes sphères de la société française en excellant à l'école. De la même manière qu'on parle de l'Amérique comme une grande démocratie, en France on entend sans cesse parler de l'éducation comme moteur de l'ascenseur social, comme le cœur de la nation. (Gumbel 11)

En même temps, son nom et son quartier de résidence le renvoient sans cesse à son statut de minoritaire, en même temps alors que son intelligence et ses lectures lui permettent de démasquer les contradictions du grand récit national qui l'entoure. Concernant ses instituteurs et professeurs, ces hussards de la République encore fortement marqués par les idéaux de la IIIe République, il note :

D'abord timidement puis très vote, je suis devenu un demi-frère exotique chez les uns, un fils adoptif chez les autres, j'entrais dans la tribu des Clovis tel un canasson dans la ville de Troie. Toujours toléré, parfois carrément adopté. Je les écoutais me parler de la France éternelle qui allait de Pagnol à Daudet, d'une France qui traversait tous les océans et tous ces gens me disaient «nous» à propos d'éclatantes victoires sur le barbare ou l'obscurantiste. À les écouter, du désert du Gobi au Sahara, de Bâton Rouge à Pondichéry, les Français dont j'étais désormais éclairent le monde avec tendresse et bonhomie. (Cherfi 17)

Car là, ou l' «imaginaire pédagogique » (pedagogical imagination) (Sachs) est encore fortement imprégné de valeurs de la III $^{\mathrm{e}}$ République, un enfant immigré sensible à ce grand récit national réactualise immédiatement un autre grand récit: celui des hussards de la République dans leur combat contre l'ignorance et l'obscurantisme. Le roman parvient à créer une véritable poétique de l'interstice, de l'entre-deux, une poétique du déchirement, et tout l'art du récit réside dans le fait que l'humour (souvent accompagné par force anacoluthes et zeugmes) met en exergue la conflagration fructueuse entre ses deux origines qui refusent de confluer dans une identité harmonieuse, hybride. Car tout comme il conteste d'être enfermé dans une identité de migrant et le discours victimaire qui l'accompagne, il est sceptique face à «l'identité trait d'union » et refuse chaque tentative de l'enfermer dans quelque identité que soit, fût-elle celle d'un "[a]rabo-beur, franco-musulman, berbéro-toulousain, gaulo-beur, franco-kabyle, maghrébo-apostat" (Cherfi 48). Sa position intermédiaire entre deux cultures fait de lui un véritable passeur de cultures malgré lui ; c'est d'ailleurs avec une violence toute particulière qu'il s'en prend à la violence machiste des grands-frères vis-à-vis des filles souvent cadenassées et à l'attitude anti-intellectualiste des caïds qui dominent la vie des jeunes dans la 
Cité. En même temps, il a bien conscience que le système scolaire ne tient pas ses promesses égalitaires et envoie les jeunes issus de l'immigration un peu rapidement vers les voies de garage menant à des parcours manuels mal-aimés.

Le roman se caractérise par une dichotomie spatialeinspirée des films sur la banlieue lesquels sont marqués par une logique spatiale : l'espace du film est clairement divisé en deux entre la banlieue, cadre familier, et cet autre espace urbain perçu comme celui de tous les privilèges ; les deux sont séparés par une ligne de démarcation très claire. Ce procédé est apparent dans le film Tout ce qui brille où deux jeunes filles demeurant à Puteaux ont pour coutume de séduire les jeunes hommes de Neuilly-sur-Seine afin de profiter de la vie de ces derniers qui brille davantage que la leur. L'on peut identifier le même procédé dans La Haine ou dans le plus récent Divines, où des frontières symboliques et souvent ethnicisées divisent l'espace en plusieurs sous-espaces. Ce même moyen peut être identifié dans Ma part de Gaulois où coexistent la banlieue, lieu d'une identité incertaine, et cet autre espace rassurant où des questionnements de nature ethnique font défaut :

J'avais envie de phrases correctement formulées, d'évoquer quelques grands esprits, Camus, Sartre, Montesquieu et Montaigne. Envie d'être invité à la table des charcuteries aveyronnaises ou de canard par ici. Du patrimoine, bordel. Pour toutes ces gourmandises, une seule adresse, celle de mon pote de terminale, Thierry. Je me délectais de sa présence. Chez lui, aucune aliénation. [...] Il ne portait aucune haine apparente, ne stigmatisait aucun groupe social ou ethnique, il flottait.

On s'est affalés dans sa chambre toute de douceurs arrangée. C'était la France entière, éternelle et dénuée de doute. Une France de meubles de bois lourds et de porcelaines séculaires, on pouvait remonter deux mille ans tranquille (sic). (Cherfi 64)

Or, même ce cadre rassurant qui lui permet de parler littérature et d'écouter, Ferré le renvoie sans cesse à son statut d'autre, de Français de la première génération. Lorsque le père de Thierry entre dans la chambre des garçons, il le félicite de sa «parfaite intégration » sur un ton des plus méprisants. Pour Magyd, «ce qui apparaissait comme une délicatesse faite homme s'est transformé en condescendance postcoloniale » (64) et, une fois de plus, on lui fait comprendre à quel point il est condamné à demeurer autre. Son désir de culture le pousse vers une sphère qui ne l'accepte que partiellement tandis que le monde dans lequel il évolue récuse les valeurs culturelles et littéraires qui lui tiennent à cœur. La déchirure qui en découle est douloureuse. Comment peut-il vivre pleinement sa part de Gaulois dès lors que, tel le Juif des Réflexions sur la question juive de Sartre, il est condamné à demeurer autre aux yeux des Français «de souche » dont il cherche désespérément à faire partie ?

Le texte de Cherfi représente ainsi une tentative d'enrichir, de renouveler, d'augmenter le roman national afin de créer une narration collective dans laquelle le plus grand nombre 
puisse se retrouver, quelle que soit l'origine ethnique de la personne. Pour ce faire, il est contraint de présenter des vérités parfois déplaisantes qui remettent en cause certaines figures mythiques de l'histoire française, dont François Mitterrand. Si, effectivement, l'héritage de Mitterrand laisse un arrière-goût amer pour certains, car il incarne comme peu d'hommes politiques les contradictions de la gauche, sa victoire a carrément terrorisé toute une génération d'Algériens dans les banlieues :

Sans rire, nos voisins se voyaient déjà dans l'obligation d'évacuer les lieux et là, s'agissait pas (sic) d'intégrer les bâtiments d'en face comme promis par la mairie, mais de faire place nette sur toute la surface du territoire mérovingien. C'était un tsunami, mais à la place des vagues, des képis hostiles allaient débouler. Moi j'en perdais mon latin, fallait-il s'inquiéter ou au contraire pousser des hourras ? J'en étais là, désorienté, confondant l'impasse et l'issue. (Cherfi 57)

En effet, l'angoisse ressentie par les Algériens tient à leur mémoire de la guerre d'Algérie qui diverge de celle des Français d'origine européenne. Bien avant le congrès d'Epernay et la création du Parti Socialiste, Mitterrand en tant que ministre de l'Outre-Mer était en charge du dossier algérien qu'il gérait avec une intransigeance particulière. Ainsi, pour les immigrés algériens son nom reste intimement lié au souvenir de la guerre. L'on voit, par ce truchement, comment deux mémoires divergentes font obstruction à un grand récit national homogène. Car, si François Mitterrand incarne pour bon nombre de Français l'espoir et l'optimisme du mois de mai, 1981, et le désir de créer une société radicalement nouvelle, une mémoire peut en cacher une autre. Le souvenir immigré diffère du grand récit franco-français. Mitterrand, le porteur d'espoir, est aussi celui qui faisait peur à la communauté immigrée en raison de ses positions intransigeantes en Algérie. Nous comprenons donc la nécessité d'établir un autre récit national qui tienne compte de la diversité des citoyens présents sur le sol français.

Véritable Bildungsroman, le texte relate comment le protagoniste parvient, à travers la musique, à réconcilier ces différentes provenances pour en faire son identité propre, marquée par des valeurs universelles. Sa multitude se fait une richesse, ses différentes histoires n'en font pas qu'une, mais coexistent pacifiquement :

Je me suis cru tiraillé, schizophrène et bancal, je ne l'étais pas plus que d'autres, sauf qu'habité par deux histoires qui se faisaient la guerre, deux familles hostiles, deux langues irrémédiablement opposées, me suis plu à être la victime expiatoire.

Comme le monde s'ouvrait à moi j'ai fait de mon fardeau des ailes, de me blessures un bouclier, de mes fêlures identitaires deux richesses dans lesquelles s'est engouffrée la seule idée qui vaille, l'universel. (Cherfi 259)

\section{Pour conclure}


Après avoir discuté la notion du roman national, espèce de béquille didactique visant à établir un lien entre nation et narration, et demandé à quel point cette grande narration collective est apte à raconter une France diversifiée, nous avons vu que les écrivains réagissent de moult manières au roman national, lequel s'inscrit dans une discussion complexe autour de ce que veut dire être Français. L'on constate d'un côté un profond attachement à la France, à sa langue et à sa littérature. Or, tous les écrivains de notre corpus plaident pour une vision plus généreuse de la francité : celle qui tienne compte de sa diversité,diversité d'abord ethnique et dont découle la nécessité d'écrire autrement l'histoire nationale. Si Jérôme Ferrari interroge un récit national purement chronologique, alors que de plus en plus nous comprenons la nature palimpseste de notre mémoire et de notre identité, Alexis Jenni nous dévoile la réalité brute des guerres coloniales et de la Résistance que la grande geste gaullienne escamotait.

Magyd Cherfi relate sa recherche de sa part de Gaulois, recherche qui le mène au cœur du grand rendez-vous manqué entre la France et ses banlieues.

Nous avons vu que la fiction, paradoxalement, permet de remettre à sa place un discours idéologique qui escamote certains faits afin d'établir un récit national, célébrant la gloire national au prix de mensonges, demi-vérités, "faits alternatifs". La discussion autour des sujets comme roman national, identité française, francité peut paraître surprenante, mais elle dévoile, du même coup, une singulière passion pour le débat, pour l'échange et pour les grandes idées, propre à une tradition française qui ne cesse de remettre en question les vérités les plus assurées et les fictions les plus enracinées.

(Timo Obergöker, Université de Chester, Royaume-Uni)

\section{Ouvrages cités :}

Boucheron, Patrick (ed.). Histoire mondiale de la France. Paris : Seuil, 2017. Imprimé.

Chambarlhac, Vincent. "Les prémisses d'une restauration? L'histoire enseignée saisie par le politique”.Histoire@Politique.Politique.Culture. Société .16 (janvier-avril 2012) : 3-16. En ligne : Histoire-politique.fr. Page consultée le 30 août 2018.

Citron, Suzanne. Le Mythe national. L'histoire de France revisitée. Paris : Éditions de l'Atelier, 1987 (reed 2016). Imprimé.

Chergi, Magyd. Ma part de Gaulois. Paris : Actes Sud, 2016. Imprimé.

Ferrari, Jérôme. Le Sermon sur la chute de Rome. Paris : Seuil, 2012. Imprimé. 
Finkielkraut, Alain. ”Les Fossoyeurs de la France”. Le Figaro, 26 janvier 2017. Imprimé.

Finkielkraut, Alain et Léa Salamé, «Stupéfiant», France 2, 20 février 2017. En ligne : https://www.youtube.com/watch?v=8sX5Mq2j6Yo. Page consultée le 30 août 2018.

Gumbel, Peter, On achève bien les écoliers. Paris : Grasset, 2010. Imprimé.

Jablonka, Ivan. L'Histoire est une littérature contemporaine. Paris : Seuil, 2017. Imprimé.

Jenni, Alexis. L'Art français de la guerre. Paris : Gallimard : 2011. Imprimé.

Jünke, Claudia. "Savoir historique et nœuds de mémoire dans L'Art français de la guerre (Alexis Jenni 2011) et Le Sermon sur la chute de Rome (Jérôme Ferrari 2012)". Revue des Sciences Humaines, janvier-mars 2016. Imprimé.

Jünke, Claudia. "Continuité et ruptures. Passé violent et conception de l'Histoire dans Où j'ai laissé mon âme de Jérôme Ferrari”. Sarah Burnautzki/Cornelia Ruhe. Coods. Chutes, ruptures et philosophie. Les romans de Jérôme Ferrari. Paris : Classiques Garnier, 2018.

Le Bris, Michel/ Jean Rouaud. Pour une littérature-monde en français. Paris Gallimard, 2007. Lindenberg, Daniel. Le rappel à l'ordre. Enquête sur les nouveau réactionnaires. Paris : Seuil, 2002.

Lukács, Georg. Der Historische Roman. Berlin (Hauptstadt der DDR): Aufbau-Verlag, 1955. Imprimé.

Martigny, Vincent. "Écrire l'Histoire de France”. Le 1, 17 mai 2017. Imprimé.

Noiriel, Gérard. À quoi sert l'identité nationale?. Paris: Agone, 2007. Imprimé.

Offenstaedt, Nicolas. "À bas le roman national" Nicolas Offenstaedt, « ̀̀ bas le roman national », L'Histoire, juillet-septembre 2009. En ligne: http://www.lhistoire.fr/bas-le-romannational. Page consultée le 30 août 2018.

Obergöker, Timo. "Le Sermon sur la chute de Rome. Une poétique de l'enracinement". Sarah Burnautzki/Cornelia Ruhe. Coods. Chutes, ruptures et philosophie. Les romans de Jérôme Ferrari. Paris : Classiques Garnier, 2018. Imprimé.

Perraud, Antoine. «La Réception d'un Goncourt » Medium 30 (2012) : 71-85. Imprimé.

Porra, Véronique, Thorsten Schüller, Danielle Dumontet, Kerstin Kloster. Eds. Les Lieux d'oubli de la Francophonie. Hildesheim : Olms, 2015. Imprimé.

Rioux, Vincent, 'L'Histoire Mondiale de la France est le reflet de son temps", L'Obs. 2 avril 2017. En ligne: https://bibliobs.nouvelobs.com/idees/20170330.OBS7323/1-histoiremondiale-de-la-france-est-le-reflet-de-son-temps.html. Page consultée le 30 août 2018.

Sachs, Leon. The Pedagogical Imagination. The Republican Legacy in Twenty-First Century Literature and Film. Lincoln/London : University of Nebraska Press, 2014. Imprimé. 
Silverman, Max. Palimpsestic Memory. The Holocaust and Colonialism in French and Francophone Film and Fiction. Londres : Berghahn Books, 2013. Imprimé.

White, Haydn. Metahistory. The Historical Imagination in Nineteenth Century Europe. Baltimore : Johns Hopkins UP, 1973.

Yale French Studies, 116/117 (2009), Turns to the right. Imprimé.

Yonnet, Marcel. Voyage au cœur du malaise français. Anti-racisme et roman national. Paris: Gallimard, 1993.

Zemmour, Eric. Mélancolie française. Paris: Albin Michel, 2010. 